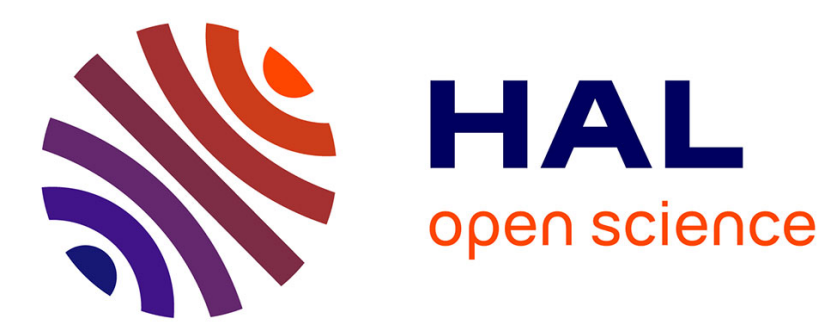

\title{
Three-dimensional stability of periodic arrays of counter-rotating vortices
}

S. Julien, Jean-Marc Chomaz, J.-C. Lasheras

\section{To cite this version:}

S. Julien, Jean-Marc Chomaz, J.-C. Lasheras. Three-dimensional stability of periodic arrays of counter-rotating vortices. Physics of Fluids, 2002, 14 (2), pp.732-743. 10.1063/1.1431246 . hal01024917

\section{HAL Id: hal-01024917 \\ https://hal-polytechnique.archives-ouvertes.fr/hal-01024917}

Submitted on 11 Sep 2014

HAL is a multi-disciplinary open access archive for the deposit and dissemination of scientific research documents, whether they are published or not. The documents may come from teaching and research institutions in France or abroad, or from public or private research centers.
L'archive ouverte pluridisciplinaire HAL, est destinée au dépôt et à la diffusion de documents scientifiques de niveau recherche, publiés ou non, émanant des établissements d'enseignement et de recherche français ou étrangers, des laboratoires publics ou privés. 


\section{AIP | Physics of Fluids}

\section{Three-dimensional stability of periodic arrays of counter-rotating vortices}

S. Julien, J.-M. Chomaz, and J.-C. Lasheras

Citation: Physics of Fluids (1994-present) 14, 732 (2002); doi: 10.1063/1.1431246

View online: http://dx.doi.org/10.1063/1.1431246

View Table of Contents: http://scitation.aip.org/content/aip/journal/pof2/14/2?ver=pdfcov

Published by the AIP Publishing

\section{Articles you may be interested in}

Effects of moderate elasticity on the stability of co- and counter-rotating Taylor-Couette flows

J. Rheol. 57, 791 (2013); 10.1122/1.4798549

Cavitation inception during the interaction of a pair of counter-rotating vortices

Phys. Fluids 24, 014107 (2012); 10.1063/1.3674299

Linear stability of a doubly periodic array of vortices to three-dimensional perturbations

Phys. Fluids 22, 054105 (2010); 10.1063/1.3415135

Three-dimensional instabilities and transient growth of a counter-rotating vortex pair

Phys. Fluids 21, 094102 (2009); 10.1063/1.3220173

Three-dimensional instability of isolated vortices

Phys. Fluids 15, 2113 (2003); 10.1063/1.1580481

\section{A|P| $\left.\right|_{\text {Applied Physics }} ^{\text {Journal of }}$}

Journal of Applied Physics is pleased to announce André Anders as its new Editor-in-Chief 


\title{
Three-dimensional stability of periodic arrays of counter-rotating vortices
}

\author{
S. Julien ${ }^{\text {a) }}$ and J.-M. Chomaz \\ Laboratoire d'Hydrodynamique (LadHyX), CNRS École Polytechnique, 91128 Palaiseau Cedex, France \\ J.-C. Lasheras \\ Department of Mechanical and Aerospace Engineering, University of California, San Diego, California 92093
}

(Received 19 January 2001; accepted 8 November 2001)

\begin{abstract}
We study the temporally developing three-dimensional stability of a row of counter-rotating vortices defined by the exact solution of Euler's equations proposed by Mallier and Maslowe [Phys. Fluids $\mathbf{5}, 1074$ (1993)]. On the basis of the symmetries of the base state, the instability modes are classified into two types, symmetric and anti-symmetric. We show that the row is unstable to two-dimensional symmetric perturbations leading to the formation of a staggered array of counter-rotating vortices. For long wavelengths, the anti-symmetric mode is shown to exhibit a maximum amplification rate at small wave numbers whose wavelengths scale mainly with the period of the row. This mode could be interpreted as due to the Crow-type of instability extended to the case of a periodic array of vortices. For short wavelengths, symmetric and anti-symmetric instability modes are shown to have comparable growth rates, and the shorter the wavelength, the more complex the structure of the eigenmode. We show that this short wavelength dynamic is due to the elliptic instability of the base flow vortices, and is well modeled by the asymptotic theory of Tsai and Widnall. The effect of varying the Reynolds number was also found to agree with theoretical predictions based on the elliptic instability. (C) 2002 American Institute of Physics. [DOI: 10.1063/1.1431246]
\end{abstract}

\section{INTRODUCTION}

The primary instability of free shear flows leads to the advection and the roll-up of the initial vorticity field into two-dimensional eddies oriented in the spanwise direction relative to the flow. The well-known mechanism of this inviscid instability is a consequence of the presence of inflexion points in the initial velocity profile. ${ }^{1,2}$ Depending on the particular configuration, the eddies organize themselves to develop extended coherent structures which have been shown to be characteristic features of free shear flows such as mixing layers, wakes or jets. These rows of vortices are usually unstable to subsequent pairing and translative instabilities. A doubling of the initial wavelength occurs through the pairing of two consecutive spanwise vortices. ${ }^{3}$ Translative instability has been identified as a secondary 3D instability of shear flows with the same periodicity as the base configuration. It precedes the transition to fully developed turbulence and is suspected to play an important role in this transition. During the evolution of the translative instability, the primary, spanwise vortices deform in a wavy fashion and a periodic array of streamwise, counter-rotating rolls appear superimposed to the primary spanwise eddies. Experimentally, this secondary 3D instability was observed by Miksad, ${ }^{4}$ Konrad $^{5}$ along with many others in mixing layers. It was also discovered to occur in the wake of a cylinder by $\mathrm{Hama}^{6}$ and later by Gerrard ${ }^{7}$ who identified a growth of the waviness of the primary vortices into "fingers of dye." Since then, many

\footnotetext{
a) Author to whom correspondence should be addressed. Telephone: 33 (0)1 $6933 \quad 36$ 72; Fax: 33 (0)1 $69 \begin{array}{llllll}33 & 30 & 30 . & \text { Electronic mail: }\end{array}$ Stephanie.Julien@ladhyx.polytechnique.fr
}

studies have shown that the appearance of this secondary structure of counter-rotating streamwise vortex pairs is also a characteristic feature of transitional wakes. As was the case with shear layers and wakes, many experimental and numerical studies have shown the existence of this secondary structure in round jets. Yule ${ }^{8}$ found azimuthal undulations of the vortex rings. Other studies have associated this undulation with the radial ejection of fluid. ${ }^{9}$

The identification of the origin of this secondary 3D instability has motivated a large body of theoretical work, most of which involves the mixing layer configuration. Pierrehumbert and Widnall, ${ }^{10}$ in 1982 , studying numerically the stability of an array of Stuart vortices identified the translative instability as a waviness of the spanwise vortices. This instability arises in the vortex cores and the ellipticity of the streamlines are responsible for its development. ${ }^{11-13}$ In 1991, Klaassen and Peltier ${ }^{14}$ focused their attention on the 3D stability in free shear layers and found not only the appearance of the core instability but also of hyperbolic modes centered in the braid region where vorticity is being withdrawn by the roll-up of the Kelvin-Helmoltz billows. A model for the generation of streamwise vortices in which the longitudinal structures could appear through the action of the plane strain existing in the braid connecting two consecutive spanwise vortices had already been proposed in 1984 by Lin and $\operatorname{Corcos}^{15}$ and later shown experimentally by Lasheras and Choi ${ }^{16}$ in 1986.

The wake configuration exhibiting two shear layers is more complex than the mixing layer since the positive and negative vorticity layers interact among themselves. Experimental studies ${ }^{17-20}$ have shown the existence of two kinds of 3D modes with distinct symmetries, characteristic of these 
wake flows. In the case of a wake behind a circular cylinder, Williamson ${ }^{19,20}$ observed that one mode, the symmetric mode (called mode A) is governed by an instability of the vortex cores and that the second one, the anti-symmetric mode (called mode B) is characterized by a wavelength four times smaller. This could be due to an instability of the hyperbolic regions.

Theoretical analyses of the secondary instability in wakes are less numerous. Robinson and Saffman ${ }^{21}$ have modeled the wake by an infinite double row of alternating sign vortices with smaller core sizes compared to both the distance between vortices and the wavelength of the instability. They showed that the staggered double row is unstable to a small wave number instability analogous to the Crow instability $^{22}$ found in a single pair of vortices. Dauxois et $a .^{23}$ have studied the two-dimensional stability of the wake model proposed by Mallier and Maslowe ${ }^{24}$ (M\&M in the following). The Mallier and Maslowe model is similar to the Stuart vortex street but consists of vortices of alternating signs aligned in a single row. Such a configuration was experimentally studied by Tabeling et al. ${ }^{25}$ who created a linear array of counter-rotating vortices generated and controlled by electromagnetic forcing. In the M\&M model, the opposite sign vortices are not staggered as for the von Kàrmàn street but form a single row of spanwise vortices. Thus, this configuration could be viewed as a model of the wake developing behind a flat plate where the initial distance between both rows of counter-rotating vortices is small. However, owing to the alignment of the vortices, this model does not contain hyperbolic points of the velocity field. Therefore, it does not represent the braid shear layer developing between two consecutive counter-rotating vortices. Dauxois et al. ${ }^{23}$ have found that there is a threshold Reynolds number beyond which the single row when subjected to a $2 \mathrm{D}$ instability develops into a staggered configuration of vortices of opposite sign.

The present paper extends the earlier studies of Dauxois et al. $^{23}$ to the analysis of 3D instability of the M\&M wake model. We will consider only instabilities with the same streamwise periodicity as the base flow. The M\&M model is described in Sec. II, the system of linearized equations that governs the stability of the M\&M solution and the numerical method used to solve the linear eigenvalue problem are described in Sec. III. In Sec. IV, we present the results obtained for three different concentrations of vortices evaluated at two different Reynolds numbers.

\section{MALLIER AND MASLOWE MODEL}

The exact 2D solution of Euler's equations proposed by $M \& \mathrm{M}^{24}$ representing a row of counter-rotating vortices is given by

$$
\psi(x, y)=\log \left(\frac{\cosh C y-C \cos x}{\cosh C y+C \cos x}\right),
$$

where $C \in[0 ; 1]$, is the concentration parameter. Velocities and length have been nondimensionalized such that the flow is $2 \pi$-periodic in $x$, and the total circulation $\Gamma$ associated with each vortex is independent of $C$ and equal to $4 \pi$.

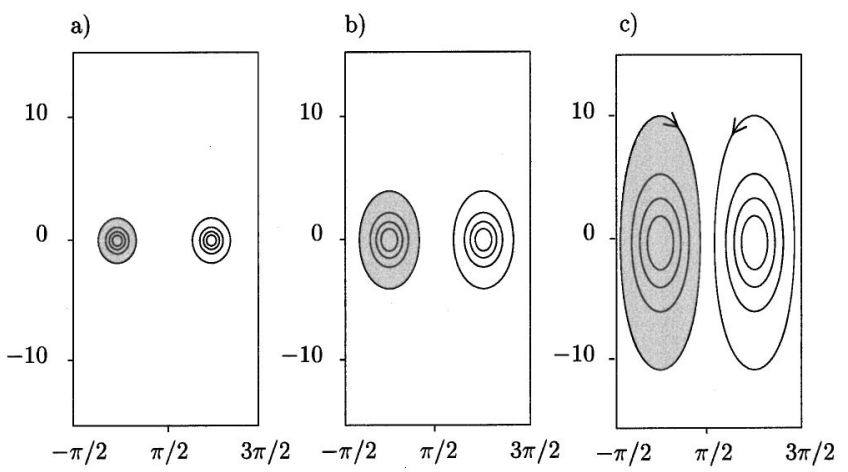

FIG. 1. Spanwise vorticity contours of the base flow in a box spanning one wavelength $\lambda_{2 \mathrm{D}}$, (a) $C=0.9$, (b) $C=0.75$, and (c) $C=0.5$. The contours values correspond to $80 \%, 60 \%, 40 \%$, and $20 \%$ of the maximum vorticity $\Omega_{\max }$ at the center of the vortex. The left vortex rotates clockwise and shaded area corresponds to negative values of vorticity $\Omega$ such that $|\Omega|$ $>0.2 * \Omega_{\max }$.

The corresponding three-dimensional velocity $\mathbf{U}$ $=(U, V, 0)$ and vorticity $\boldsymbol{\Omega}=(0,0, \Omega)$ fields, with $\Omega$ $=-\Delta \psi$, are given by

$$
\begin{aligned}
& U(x, y)=\frac{2 C^{2} \sinh C y \cos x}{\cosh ^{2} C y-C^{2} \cos ^{2} x}, \\
& V(x, y)=-\frac{2 C \sin x \cosh C y}{\cosh ^{2} C y-C^{2} \cos ^{2} x}, \\
& \Omega(x, y)=\frac{\left(1-C^{2}\right)}{2} \sinh (2 \psi) .
\end{aligned}
$$

The value of the parameter $C$ controls the concentration of the vortices. The limiting value $C=1$ corresponds to point vortices while $C=0$ represents a fluid at rest $(\psi(x, y)=0)$. The larger the value of $C$, the more concentrated the vortex cores are. This is shown in Fig. 1 where the iso-contours of the vorticity field in one period for $x \in[-\pi / 2 ; 3 \pi / 2]$ are plotted for three different values of the concentration parameter $C=0.9, C=0.75$, and $C=0.5$. It is important to point out that the iso-vorticity contours are elliptical with their major axis oriented in the vertical direction. In addition, the ellipticity of the vortex cores increases with decreasing values of the concentration parameter.

For each value of the concentration parameter $C$ studied, one may define different length scales characterizing the core size of the vortices. We chose the length scale, denoted by $a$, obtained from the first moment of the spanwise base flow vorticity $\Omega$ (Ref. 26)

$$
a=\frac{1}{\Gamma} \int_{-\pi / 2}^{+\pi / 2} \int_{-\infty}^{+\infty}\left(x^{2}+y^{2}\right)^{1 / 2} \Omega(x, y) d x d y .
$$

Moreover, we define the aspect ratio $\theta=d / c$ that characterizes the ellipticity of the streamline around the center of the vortex. $c$ and $d$ are obtained by expanding the vorticity function about $(x, y)=(0,0)$ where it achieves the maximum value $\Omega_{\max }$

$$
\Omega(x, y)=-\Omega_{\max }\left(1-\frac{x^{2}}{c^{2}}-\frac{y^{2}}{d^{2}}+\vartheta\left(x^{2}, y^{2}\right)\right),
$$


TABLE I. Vortex core size and aspect ratio parameter.

\begin{tabular}{lccc}
\hline \hline \multicolumn{1}{c}{$a$} & $a$ & $\theta$ & $\Omega_{\max }$ \\
\hline 0.9 & 0.68 & 1.11 & 17.15 \\
0.75 & 1.17 & 1.33 & 5.36 \\
0.5 & 2.15 & 2 & 1.67 \\
\hline \hline
\end{tabular}

where $\vartheta\left(x^{2}, y^{2}\right)$ indicates that we are neglecting terms smaller than $x^{2}$ or $y^{2}$ when $x$ and $y$ goes to zero.

The estimated vortex core size and the aspect ratio given by the parameters defined above are gathered in Table I. Note that the larger the value of the concentration parameter $C$, the smaller the core size and the ellipticity. As the core size decreases, the maximum vorticity increases to conserve the circulation.

\section{PERTURBATION EQUATION AND NUMERICAL PROCEDURE}

Since the $M \& M^{24}$ solution is uniform along the $z$ axis, we consider a general linear perturbation of the form

$$
[\widetilde{\mathbf{u}}, \widetilde{\boldsymbol{\omega}}, \widetilde{\mathbf{p}}](x, y, z, t)=[\mathbf{u}, \boldsymbol{\omega}, \mathbf{p}](x, y, t) e^{i k z}+\text { c.c. },
$$

where $\widetilde{\mathbf{u}}, \widetilde{\boldsymbol{\omega}}=\boldsymbol{\nabla} \wedge \widetilde{\mathbf{u}}$ and $\widetilde{\mathbf{p}}$ are, respectively, the 3D velocity, vorticity and pressure perturbations and $k$ is the wave number in the $z$-spanwise direction. $\mathbf{u}, \boldsymbol{\omega}$ are complex three component vector fields, c.c. in Eq. (7) denotes the complex conjugate of the first term in the right-hand side. The evolution of the perturbation is governed by the Navier-Stokes equations linearized around the base flow defined by $U$ and $\Omega(2$, $3,4)$

$$
\operatorname{div} \widetilde{\mathbf{u}}=0
$$

and

$$
\frac{\partial \widetilde{\mathbf{u}}}{\partial t}+\mathbf{\Omega} \wedge \widetilde{\mathbf{u}}+\widetilde{\boldsymbol{\omega}} \wedge \mathbf{U}=-\nabla[\widetilde{\mathbf{p}}+\widetilde{\mathbf{u}} \cdot \mathbf{U}]+\nu \Delta \widetilde{\mathbf{u}},
$$

where $v$ is the viscosity. Since $\Gamma=4 \pi$ for the M\&M solution, the Reynolds number based on the circulation $\mathrm{Re}$ $=\Gamma / 4 \pi v$ is always $\operatorname{Re}=1 / v$.

As mentioned before, the base flow defined in Sec. II is an exact solution of the inviscid Navier-Stokes equations. We will analyze the stability properties of this flow in the presence of viscosity by assuming that the viscous diffusion of the base flow is balanced by a "body force" $(-v \Delta \mathbf{U})$. This is commonly done (see the viscous theory of parallel shear flows, p. 153 in Ref. 3) when studying the stability under the full Navier-Stokes equations of a solution of Euler's equations. Note that, consistent with this approach, the term $v \Delta \mathbf{U}$ does not appear in this Navier-Stokes formulation (9). When comparing to experiments, this approximation is expected to be quite reasonable if the growth rate of the $3 \mathrm{D}$ instabilities is large compared to the viscous damping of the base flow.

The perturbations $\mathbf{u}, \boldsymbol{\omega}$, and $\mathbf{p}$ are expressed in Fourier space via a 2D complex Fourier transform

$$
\begin{aligned}
{[\mathbf{u}, \boldsymbol{\omega}, \mathbf{p}](x, y, t)=} & \iint[\hat{\mathbf{u}}, \hat{\boldsymbol{\omega}}, \hat{\mathbf{p}}] \\
& \times\left(k_{x}, k_{y}, t\right) * e^{i\left(k_{z} x+k_{y} y\right)} d k_{x} d k_{y}
\end{aligned}
$$

where $k_{x}$ and $k_{y}$ are the horizontal components of the wave vector $\mathbf{k}_{t}=\left(\mathbf{k}_{x}, \mathbf{k}_{y}, \mathbf{k}\right)$.

In spectral space, the Navier-Stokes equations take the form

$$
\frac{\partial \hat{\mathbf{u}}}{\partial t}=P\left(\mathbf{k}_{t}\right)[\mathbf{u} \wedge \widehat{\boldsymbol{\Omega}+\mathbf{U}} \wedge \boldsymbol{\omega}]-v \mathbf{k}_{t}^{2} \hat{\mathbf{u}}
$$

where $P\left(\mathbf{k}_{t}\right)$ is the projection operator onto the space of solenoidal fields (i.e., $P_{i j}=\delta_{i j}-k_{i} k_{j} / \mathbf{k}_{t}^{2}$ ).

The linear eigenvalue problem that corresponds to seeking exponentially growing solutions of (11) is solved using an iterative power method (see Ref. 27 for details). For this purpose, Eq. (11) is integrated in time starting from random initial conditions. Since we are considering only perturbations that preserve the periodicity of the base flow here, the integration domain is chosen to be equal to one wavelength of the base flow, (i.e., $x \in[\pi / 2,3 \pi / 2]$ ). If other Floquet multipliers were of interest, the box size should have been adapted (that is, if one were considering the pairing mode, the size of the integration domain should be doubled in $x$ ). The perturbation is assumed to be periodic in the $y$ direction. To avoid boundary effects, the box size in the $y$ direction is chosen wide. The size $L_{y}=4 \pi$ has been found to be sufficiently large since the results of our computations remain unaffected by increasing the $y$-dimension beyond $4 \pi$.

The numerical integration code, written in Cartesian coordinates with periodic boundary conditions in $x$ and $y$, is based on a pseudo-spectral method. Since all the details of the numerical procedure and the validation of this code are given in Billant et al., ${ }^{28}$ we will omit its description here. The linearized advection term, i.e., the term in brackets on the right-hand side of (11) is evaluated in the physical space via successive Fourier transforms in the $x$ and $y$ directions, and the $2 / 3$ rule is applied for de-aliasing. The viscous term $\left(\mathbf{k}_{t}^{2} v \hat{\mathbf{u}}\right)$ is exactly integrated in the spectral space. This means that the time evolution is computed on $\hat{\mathbf{u}}\left(k_{x}, k_{y}, t\right) e^{\mathbf{k}_{t}^{2} v \mathbf{t}}$ instead of $\hat{\mathbf{u}}\left(k_{x}, k_{y}, t\right)$ as fully described in Ref. 28. This time evolution is realized in the spectral space using the second-order Adams-Bashforth scheme.

For each value of the transverse wave number $k$, the perturbation velocity field is initialized with a divergencefree white noise. The integration of (11) is carried on in time long enough for the most unstable mode to dominate. This method gives only access to the most unstable mode. When $t \rightarrow \infty$, the velocity $\mathbf{u}(x, y, t)$ tends to a solution of the form: $\mathbf{A}(x, y) \exp (\sigma t)$, where $\mathbf{A}(x, y)$ is the $3 \mathrm{D}$ complex eigenfunction and $\sigma$ is the leading eigenvalue. The real part of $\sigma$ represents the temporal growth rate, and the imaginary part the frequency of the most amplified mode. To speed up the convergence of our method, the eigenmode found at a close value of $k$ may be used instead of white noise to initialize the computation. 
The growth rate is computed from the kinetic-energy $E$ of the perturbation integrated over the whole computational domain

$$
E=\iint \mathbf{u u}^{*} d x d y,
$$

* denoting the complex conjugate

$$
\sigma_{r}=\lim _{t \rightarrow \infty} \frac{1}{2} \frac{d \ln E}{d t} .
$$

The leading eigenmode is approximated by the perturbation obtained at the last time-step of the simulation. The convergence criteria is such that $1 / \sigma_{r} d \sigma_{r} / d t$ is less than $10^{-3}$ at the last time steps of the computation.

For the smaller concentration parameters, $C=0.5$ and 0.75 , the computations are performed on a Cartesian mesh consisting of $64 \times 128$ collocation points with a mesh size in the $x$ and $y$ direction equal to $\delta_{x}=\delta_{y} \simeq 0.098$. A resolution of $128 \times 256$ collocation points with $\delta_{x}=\delta_{y} \simeq 0.049$ has been used for the case $C=0.9$. The integration time step is $\delta_{t}$ $=0.01$ for $C=0.5$ and $C=0.75, \delta_{t}=0.001$ for $C=0.9$. Considering that the maximum velocity of the base flow increases with the concentration parameter $C$, this choice of time steps allows the Courant-Friedrich-Levy condition ( $\Delta x / \Delta t>U_{\max }$ ) to be satisfied by one order of magnitude in all three concentration cases considered here.

As demonstrated by Robinson and $\operatorname{Saffman}^{21}$ for the more general case of a staggered double row of vortices, the instability modes can be separated into two families. In our case, since the base state vorticity is symmetric when $(x, y)$ $\rightarrow\left(x+\lambda_{2 \mathrm{D}} / 2,-y\right)$, the symmetry of both mode families simplifies to:

$$
\begin{aligned}
& \text { the symmetric mode (also called the varicose } \\
& \text { mode } \left.{ }^{17,18}\right) \text { with } \\
& {\left[u_{x}, u_{y}, u_{z}\right](x, y, t)=\left[u_{x},-u_{y}, u_{z}\right]\left(x+\lambda_{2 \mathrm{D}} / 2,-y, t\right),} \\
& \text { or equivalently } \\
& {\left[\omega_{x}, \omega_{y}, \omega_{z}\right](x, y, t)=\left[-\omega_{x}, \omega_{y},-\omega_{z}\right]\left(x+\lambda_{2 \mathrm{D}} / 2,-y, t\right),}
\end{aligned}
$$

(i) and the anti-symmetric mode (also called the sinuous mode $\left.{ }^{17,18}\right)$, with

$$
\left[u_{x}, u_{y}, u_{z}\right](x, y, t)=\left[-u_{x}, u_{y},-u_{z}\right]\left(x+\lambda_{2 \mathrm{D}} / 2,-y, t\right),
$$

or equivalently

$$
\left[\omega_{x}, \omega_{y}, \omega_{z}\right](x, y, t)=\left[\omega_{x},-\omega_{y}, \omega_{z}\right]\left(x+\lambda_{2 \mathrm{D}} / 2,-y, t\right) .
$$

These denominations follow those given by Robinson and Saffman. ${ }^{21}$ It should be emphasized that it corresponds to the symmetry of the perturbation field $u_{z}$ (14) and (16). For example, the anti-symmetric mode is such that $u_{x}$ and $u_{z}$ are anti-symmetric whereas $u_{y}$ is symmetric under the transformation $(x, y) \rightarrow\left(x+\lambda_{2 \mathrm{D}} / 2,-y\right)$.
These symmetries have been imposed on the initial random condition or forced at each time step on the velocity field $u$. With both methods, the results of our iterative power method are identical.

To investigate the effect of viscosity, we have studied two different Reynolds numbers, $\operatorname{Re}=(400,5000)$ for three distinct concentration parameters of the base flow vortices $C=(0.5,0.75,0.9)$. For both symmetric and anti-symmetric families, the growth rates $\sigma_{r}$ have been computed as a function of the spanwise wave number $k$ in the range $[0 ; 9]$ and the associated eigenfunctions determined. In the following, the growth rate $\sigma_{r}$ will be denoted by $\sigma$.

\section{RESULTS}

\section{A. Evolution of the growth rate}

\section{Anti-symmetric modes}

Figure 2 presents the growth rate curves $\sigma$ as a function of the spanwise wave number $k$ for the anti-symmetric perturbation. Each diagram corresponds to a specific concentration, (a) $C=0.9$, (b) $C=0.75$, (c) $C=0.5$, the curve defined by open symbols corresponding to $\mathrm{Re}=5000$ and the curve with filled symbols to $\operatorname{Re}=400$. For $C=0.9$ [Fig. 2(a)], the base flow vortices are strongly concentrated and well separated whereas they are diffused and not distinctly separated for $C=0.5$.

For $C=0.9$, the growth rate of the anti-symmetric mode, stable at $k=0$, exhibits a well defined peak at low wave numbers with the maximum located at $k_{\max }=0.4$. The peak is confined to the band $[0 ; 0.9]$. Hereafter, we will call it the Crow instability mode since, as explained in Sec. IV B 1, it is the extension to an infinite row of vortex pairs of the Crow instability defined for a single vortex pair. This instability will be labeled Cr. For larger wave numbers, the growth rate presents two other maxima. These two maxima define two different regions, labeled as $E_{1}$ and $E_{2}$ in Fig. 2(a), where the curves are "bell-shaped" for both Reynolds numbers. These modes will be called elliptic modes.

For the concentration parameter $C=0.75$, Fig. 2(b), we obtained similar behavior. The Crow instability peak is visible in the range $[0 ; 0.9]$ with a maximum amplification rate lower than that computed for $C=0.9$, but still occurring at the value of $k_{\max }=0.4$. For $\operatorname{Re}=5000$, four other peaks (as opposed to the two found for $C=0.9$ ) are clearly visible defining elliptic instability modes $\left(E_{1}, E_{2}, E_{3}, E_{4}\right)$. Their maximum growth rates are smaller than those found for the largest concentration parameter $C=0.9$. The first and second maxima also occur at a lower wave number for $C=0.75$ than for $C=0.9$.

For the smaller concentration parameter $C=0.5$, Fig. 2(c), the Crow instability peak is not visible around $k=0.4$ but the curve still presents a clear breaking in curvature at $k=0.9$ where the growth rate slope changes radically. For large wave numbers, the growth rate does not exhibit any peaks but the regions $\left(E_{1}, E_{2}, E_{3}, E_{4}\right)$ shown in Fig. 2(c) were nevertheless defined using a criterion which will be explained later. 
a)

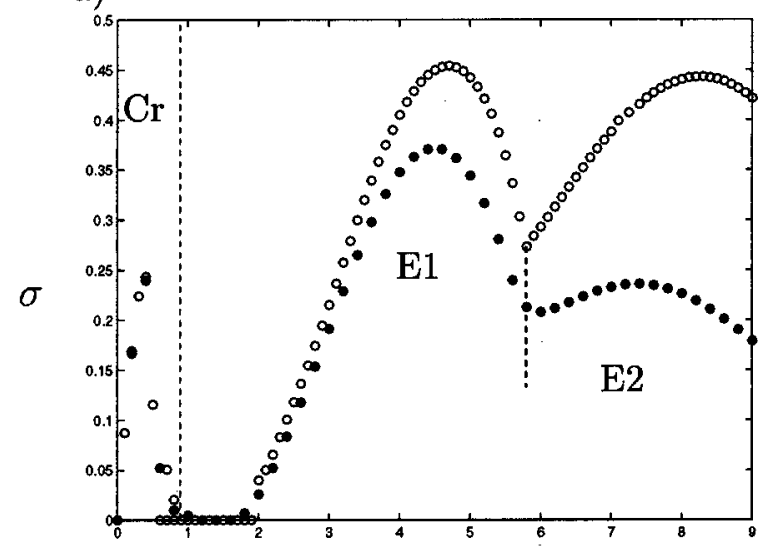

k

b)

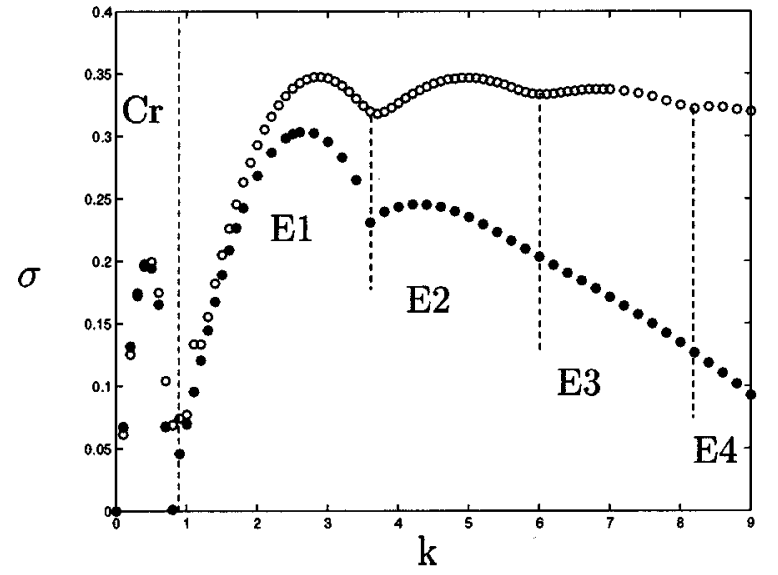

c)

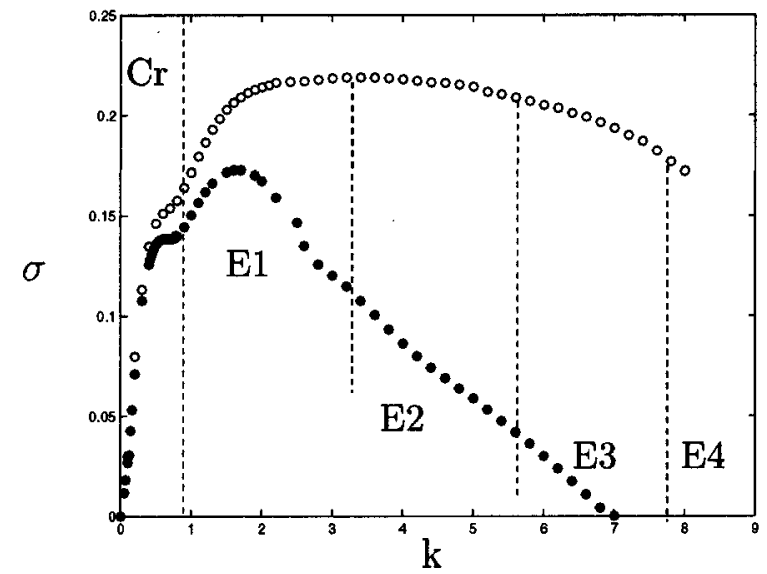

FIG. 2. Growth rate curves of the anti-symmetric mode as a function of the transverse wave number $k$ for both Reynolds numbers $\mathrm{Re}=400$ (filled symbols) and $\mathrm{Re}=5000$ (open symbols), (a) $C=0.9$, (b) $C=0.75$, (c) $C=0.5$.

\section{Symmetric modes}

Subjected to symmetric perturbations, the M\&M configuration is unstable at $k=0$ (Fig. 3) whichever concentration $C$ is studied. The growth rate of this $2 \mathrm{D}$ mode depends strongly on $C$ : It decreases as $C$ increases, tending to zero when the limit of point vortices is approached. Unlike the anti-symmetric case, the symmetric growth rate curves do a)

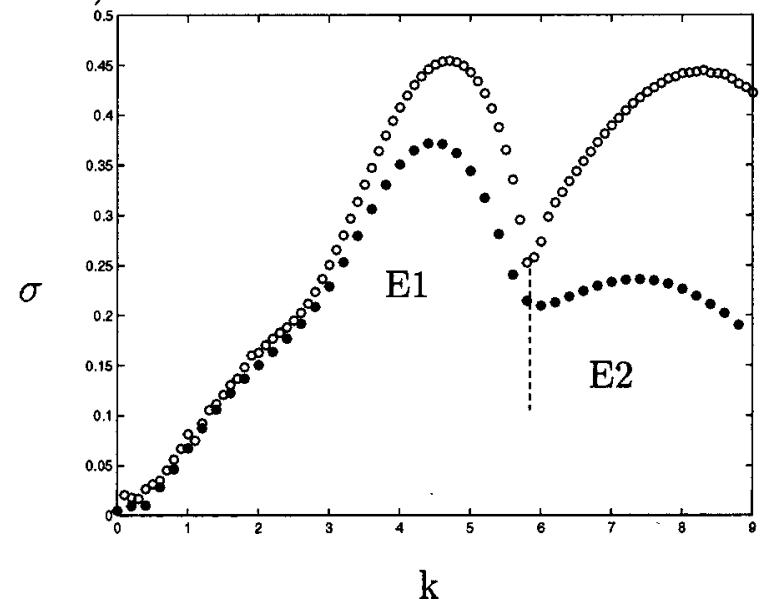

b)

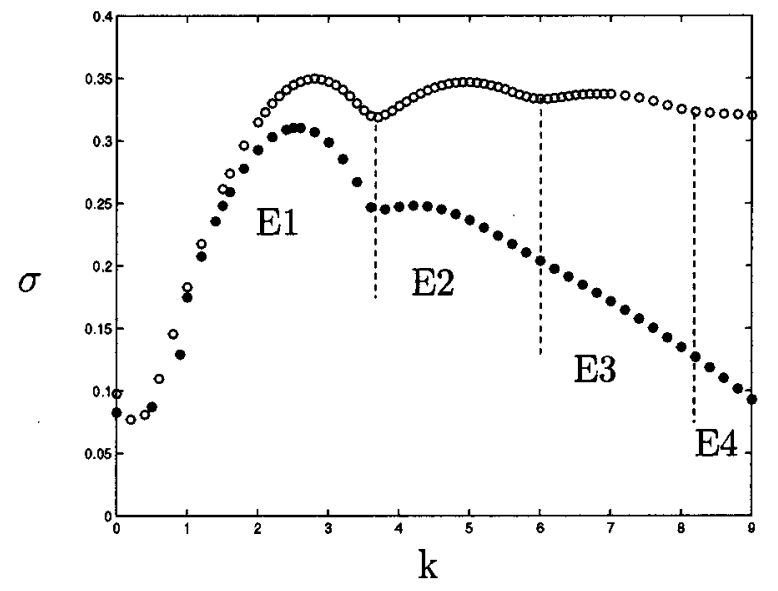

c)

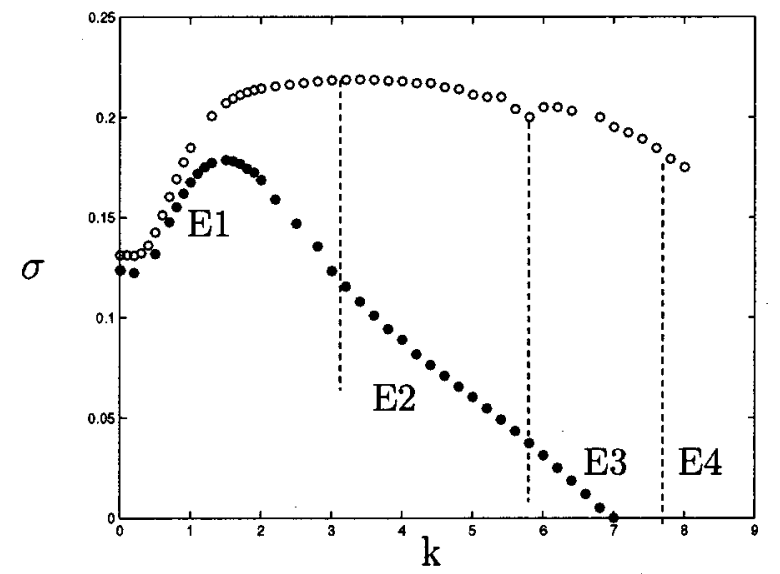

FIG. 3. Growth rate curves of the symmetric mode as a function of the transverse wave number $k$ for both Reynolds numbers $\mathrm{Re}=400$ (filled symbols) and $\operatorname{Re}=5000$ (open symbols) (a) $C=0.9$, (b) $C=0.75$, (c) $C=0.5$.

not present a peak at low wave number. At large wave numbers, the growth rate of the symmetric mode behaves similarly to its anti-symmetric counterpart. The growth rates present a sequence of "bell-shaped" sections with different maxima. The values of the maxima and the associate wave numbers, as well as the elliptic regions $\left(E_{1}, E_{2}\right)$ for $C=0.9$ and $\left(E_{1}, E_{2}, E_{3}, E_{4}\right)$ for $C=0.75$, are identical to the antisymmetric ones. 
TABLE II. Table comparing the numerically computed cut-off wave numbers $k_{c}$ at $\operatorname{Re}=400$ to the value $k^{*}$ predicted by the viscous elliptic instability theory for each concentration $C$ studied.

\begin{tabular}{lcc}
\hline \hline$C$ & $k_{c}$ & $k^{*}$ \\
\hline 0.9 & 12.6 & 13.1 \\
0.75 & 11 & 11.5 \\
0.5 & 7 & 9 \\
\hline \hline
\end{tabular}

\section{Effect of viscosity}

The effect of Reynolds number on the growth rate of the instability may be analyzed by comparing curves obtained for $\operatorname{Re}=5000$ (open symbols) to those computed for $\mathrm{Re}$ $=400$ (filled symbols). It is clear that for the three values of $C$ studied here, the growth rates in the more viscous case $\operatorname{Re}=400$ are always smaller than for $\mathrm{Re}=5000$. Furthermore, the larger the $k$, the larger the viscous damping. It should be noted that for $\mathrm{Re}=400$, wave numbers $k$ larger than a particular threshold value $k_{c}$ are totally stabilized. This threshold wave number $k_{c}$ increases with the value of the concentration parameter $C$ (Table II). Comparison between Re $=5000$ and $\mathrm{Re}=400$ may be carried out anticipating the elliptic nature of the instability at large $k$. Landman and Saffman, ${ }^{29}$ studying the 3D stability of an elliptical 2D flow with uniform constant vorticity in the viscous case, have shown that the growth rate $\sigma(k)$ is such that

$$
\sigma(k)=\sigma_{\infty}(k)-v k^{2}\left[1+\frac{1}{2}\left(\theta^{2}-1\right) \sin ^{2} \alpha\right],
$$

where $\sigma_{\infty}(k)$ is the inviscid growth rate, $v$ the viscosity, $k$ the wave number, $\theta$ the aspect ratio of the elliptical streamlines, and $\alpha$ the inclination angle of the wave vector to the spanwise direction.

When the ellipticity is small, the leading order of Eq. (18) gives

$$
\sigma(k)=\sigma_{\infty}(k)-v k^{2} .
$$

Although the inviscid growth rate $\sigma_{\infty}(k)$ is unknown, we may approximate $\sigma_{\infty}(k)$ by the growth rate $\sigma_{5000}(k)$ numerically obtained at $\operatorname{Re}=5000$ for the same $k$. Then one may predict the cut-off values $k^{*}$ for the viscous stabilization at $\operatorname{Re}=400$ as given by the implicit equation $\sigma_{5000}\left(k^{*}\right)$ $-v k^{* 2}=0$ where $v$ is the viscosity corresponding to Re $=400$. The predicted cut-off values $k^{*}$ are given in Table II and compare very favorably with the computed cut-off values $k_{c}$.

\section{B. Modal structures}

The above study of the growth rate curves has allowed us to identify several instability regions which were found to depend not only on the symmetry characteristics of the mode, but also on the order of magnitude of the wave number. For all combinations of values of the concentration parameter $C$ and the Reynolds numbers we have shown that regardless of the symmetry of the mode, the growth rates diagrams are markedly different in two wave number ranges. At low $k$, the anti-symmetric and symmetric modes behave distinctly, while at large $k$ both growth rates behave similarly.

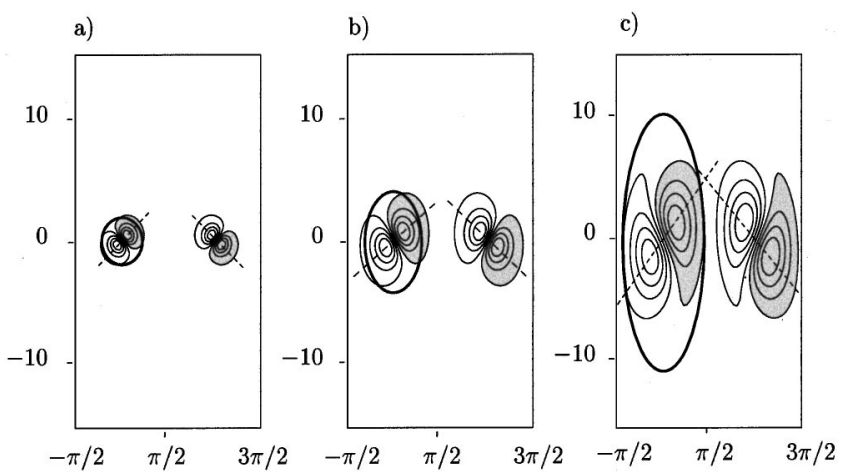

FIG. 4. Spanwise isovorticity contours of the Crow anti-symmetric mode, $k=0.4$, at $\operatorname{Re}=400$, (a) $C=0.9$, (b) $C=0.75$, and (c) $C=0.5$. The heavy contour marks the base flow vortex core since it represents isovorticity contour of the spanwise base flow vorticity corresponding to $0.2 \Omega_{\max }$. The dashed lines indicate the orientation of the perturbation dipole. Shaded area corresponds to negative values of vorticity $\Omega$ such that $|\Omega|>0.2 * \Omega_{\max }$.

In the present section, we shall demonstrate that the behavior of the modes in each of these wave number regions is characterized by a particular eigenmode structure with a distinct instability mechanism.

\section{The periodic Crow instability}

The spanwise vorticity distribution corresponding to the eigenmode at $k=0.4$ for which the maximum amplification rate of the Crow instability was calculated is plotted in Fig. 4. In the cases $C=0.9$ and $C=0.75$, Figs. 4(a) and 4(b), the spanwise vorticity consists of two opposite sign perturbations, centered on each base flow vortex and inclined at $47^{\circ}$ with respect to the $y$ axis. The heavy contour in Figs. 4(a) and 4 (b) corresponds to the iso-vorticity contour $0.2 \Omega_{\max }$ of the base flow and is indicative of the size of the vortex core. Comparing the eigenmode to the core of the vortex indicates that it affects the whole vortex, inducing an out-of phase undulation of each base vortex in the planes tilted at $47^{\circ}$ with respect to the $y$ axis. This out-of-phase undulation induces the vortices to alternatively approach or recede from one another as one moves either in the positive or negative $y$ directions (Fig. 5).

For the value of the concentration parameter $C=0.5$, Fig. 4(c), the perturbation is seen to have the same shape, but the perturbation dipoles are inclined at $50^{\circ}$ to the vertical $y$ axis.

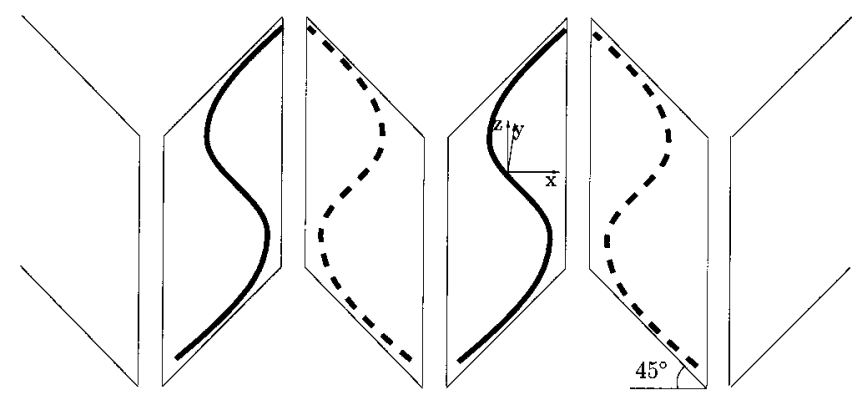

FIG. 5. Scheme of periodic Crow instability. The plain vortices and the dashed vortices rotate in opposite directions. 
The eigenmode structure described above is similar to the perturbation mode of a single dipole known as the Crow instability (Fig. 8 of Crow's study ${ }^{22}$ ). However, it should be pointed out that there are considerable differences between the modes described above and those studied by Crow. In fact, Crow's theory is concerned with just a single pair of counter-rotating vortices with uniform vorticity, and is valid only in the limit where the vortex cores are smaller than the separation between eddies. In contrast, the M\&M solution represents an infinite row of counter-rotating pairs of vortices with distributed vorticity. Only when the concentration parameter $C$ is close to unity can the core of the vortices be assumed to be small compared to the distance between vortices.

Robinson and Saffman ${ }^{21}$ have extended Crow's study ${ }^{22}$ to vortex streets. Following their convention, Crow's instability is anti-symmetric (16) and (17) whereas for a single dipole, this instability is labeled symmetric since it refers to a different symmetry (i.e., $x \rightarrow-x$ ). For a staggered double row of vortices Robinson and Saffman have shown that this 3D long-wavelength instability is dominant over a large range of the ratios $\kappa=h / \lambda_{2 \mathrm{D}}$, where $h$ is the distance between the two rows of counter-rotating vortices $(h=0$ so that $\kappa=0$ for the M\&M model) and $\lambda_{2 \mathrm{D}}$ is the $2 \mathrm{D}$ wavelength defining the periodicity of each row. For very concentrated vortices $a / \lambda_{2 \mathrm{D}}=0.1$ ( $a$ is the vortex core size), they predicted that the most amplified wave number is 0.36 with a growth rate equal to 0.25 (Fig. 5 of Ref. 22). For the largest concentration parameters $C=0.9$ where the vortex core size is $a / 2 \pi=0.05$, we obtain a maximum at $k=0.4 \pm 0.05$ with a maximum growth rate $\sigma_{\max }=0.25$. For $C=0.75, a / 2 \pi=0.1$ and the maximum occurs at $k=0.4 \pm 0.05$ with $\sigma_{\max }=0.21$. Agreement with Robinson and Saffman predictions is excellent, further confirming that the mode dominating at small values of $k$ corresponds to a Crow-type mode.

Acknowledging that in the M\&M configuration, a periodic row of a pair of counter-rotating vortices with distributed vorticity is radically different from Crow's configuration, a single pair of uniform counter-rotating vortices, we compare in Fig. 6 the growth rate obtained numerically for $\mathrm{Re}=5000$ and the growth rate predicted by Crow's theory ${ }^{22}$ by taking the distance between vortices $b$ equal to $\pi$ as in our case and by using the estimated vortex core size $a$.

Following Crow's work, the growth rate $\sigma$ and the wave number $k$ of the M\&M solution are respectively re-scaled by $\Gamma / 2 \pi b^{2}$ and by $b$ on Fig. 6 .

It is quite remarkable that for the largest concentration parameter studied $C=0.9$, Crow's theory for small wave number $k b$ predicts quite correctly our computed growth rates at small $k b$ (Fig. 6). Of course, at larger $k b$, the asymptotic theory of Crow is not valid but it still appears to predict the behavior of our growth rate curve. For the concentration $C=0.75$, the agreement is less precise since the ratio of the vortex size to the vortex spacing is too large and invalidates the asymptotic theory. Nevertheless, the correct trends and order of magnitude for the growth rate curve appear to be well predicted by the theory. Surprisingly, for $C$ $=0.5$, where the core of the vortices is so large that they nearly touch each other, the theory, valid for well-separated

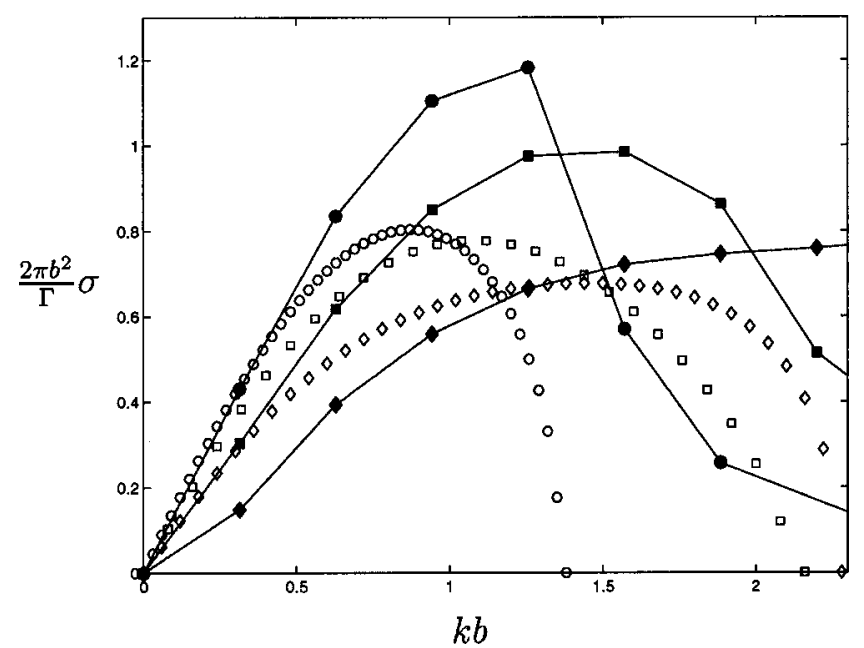

FIG. 6. Comparison between the anti-symmetric growth rate peak and Crow's predictions. Filled symbols are relative to our numerical calculations and open symbols to Crow's predictions. The circles correspond to $C$ $=0.9$, the squares to $C=0.75$ and the diamonds to $C=0.5$.

scales between vortex core size and vortices distance, still describes the general trend reasonably well.

\section{The two-dimensional instability}

In the $2 \mathrm{D}$ case, we have shown that the M\&M flow is unstable only to a symmetric mode.

The corresponding eigenfunction is given in Fig. 7. In this case, the perturbation is concentrated into two opposite sign perturbations, each one being centered on one base flow vortex and slightly tilted from the $y$ axis. As we have done in the previous cases, the isoline $0.2 \Omega_{\max }$ of the base flow vorticity is also shown in Fig. 7 as a heavy line to indicate the vortex core of positive sign. It may be observed that the perturbation is intense inside the whole vortex. This perturbation corresponds to a uniform displacement (indicated in Fig. 7 by arrows) of the base vortices opposite in the $y$ direction between the positive and the negative vortices. This mode, leading to two staggered rows of counter-rotating vortices, has already been described by Dauxois et al. (Fig. 4 of Ref. 24). The effect of Reynolds number has also been studied by Dauxois et al. They found that the growth rate asso-

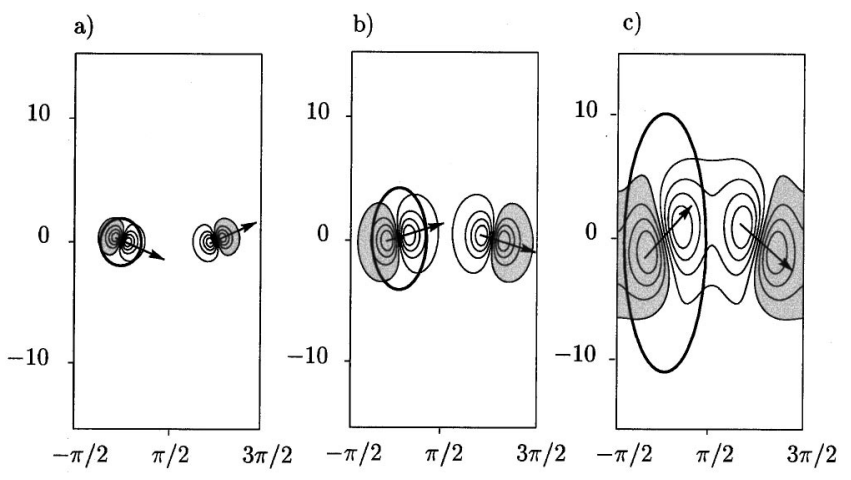

FIG. 7. Same representation as Fig. 4 for the eigenmode of the 2D instability presented as spanwise vorticity contours. The arrows represent the displacements undergoing by the base vortices. 


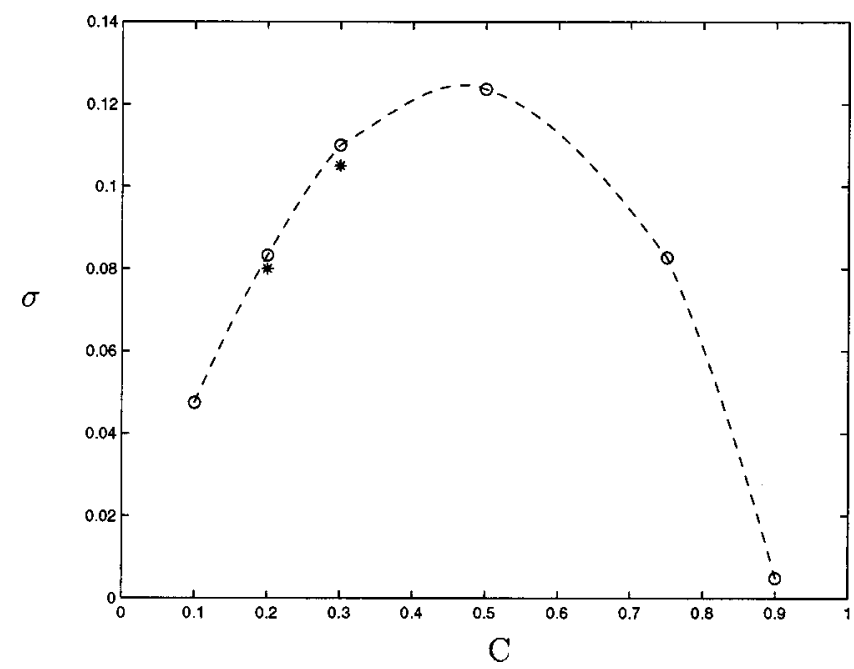

FIG. 8. Growth rate of the 2D mode $(k=0)$ as a function of $C$ for $\operatorname{Re}$ $=400$. The stars symbols refer to the results of Dauxois et al.

ciated to this mode depends on the Reynolds number and that for sufficiently small Reynolds number (of order 1 for $C=0.5$ ), the base configuration remains stable. The slight damping of the growth rate observed when the Reynolds number decreases (see Fig. 3 in the previous section) is then consistent with their result. However, we have not considered low enough Reynolds number to observe stabilization.

Figure 8 presents the computed growth rates of the 2D instability as a function of the concentration $C$ for the case of $\mathrm{Re}=400$. In order to compare to Dauxois et al., who restricted themselves to cases with $C<0.5$, the $2 \mathrm{D}$ instability mode has been computed for $C=0.1, C=0.2$, and $C=0.3$. As shown in Fig. 8 our results are in excellent agreement with Dauxois et al. whose results are plotted as stars in the figure. For $C$ lower than 0.5 , the growth rate of the $2 \mathrm{D}$ mode is seen to increase with the concentration parameter. However, for $C$ larger than 0.5 , the trend reverses and the growth rate decreases, Fig. 8. The decrease of $\sigma$ with the concentration is to be expected since for $C=1$, the vortices are infinitely concentrated and the point vortex stability theory ${ }^{30}$
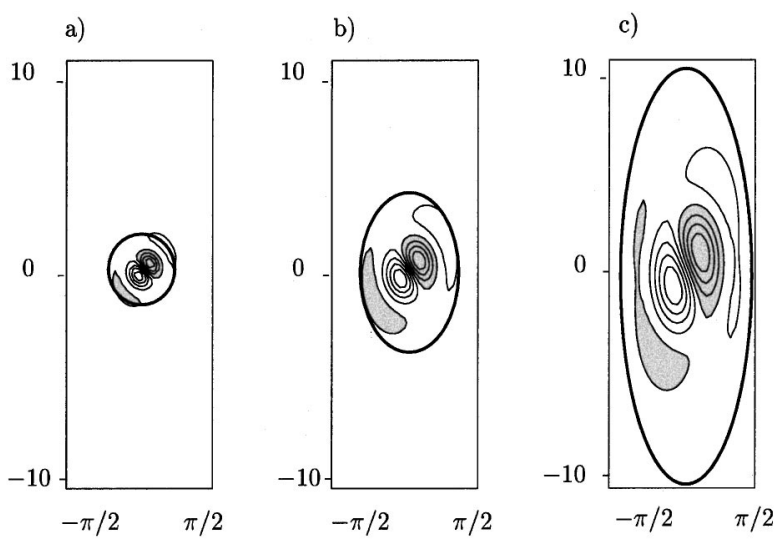

FIG. 9. Spanwise vorticity contours of the elliptic anti-symmetric mode in band $\mathrm{E} 1$ at $\mathrm{Re}=5000$, (a) $C=0.9, k=4.7$, (b) $C=0.75, k=2.9$, (c) $C$ $=0.5, k=1.6$. a)

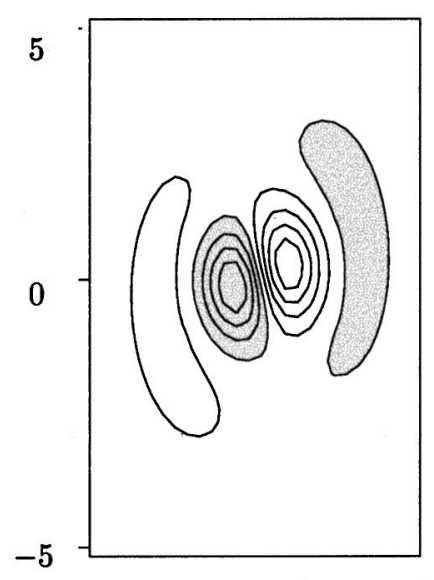

b)

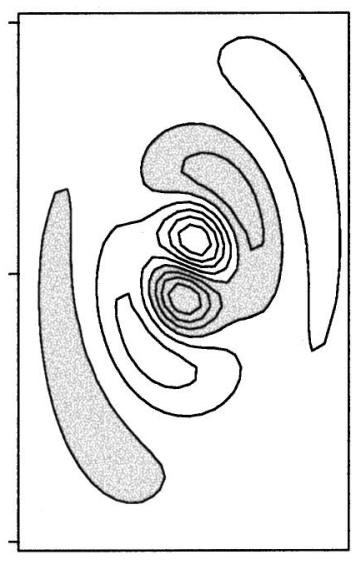

c)

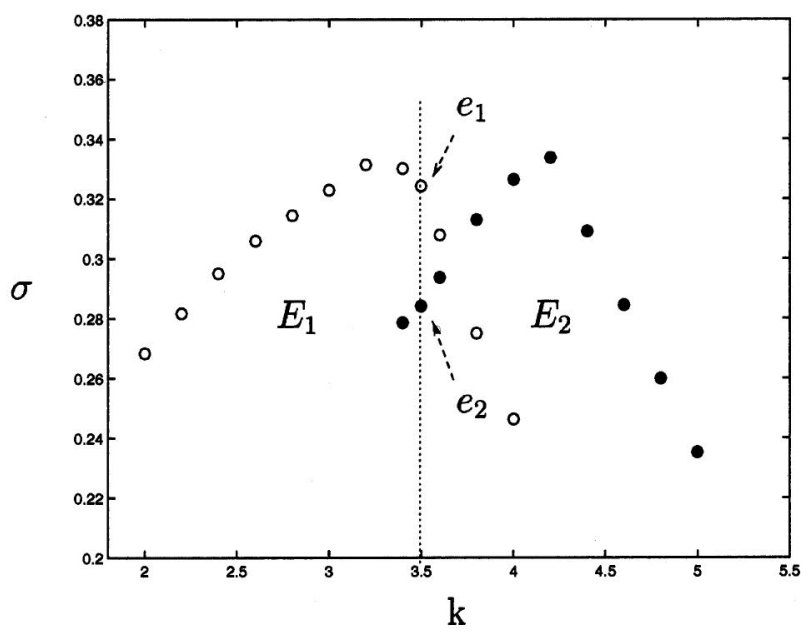

FIG. 10. (a) $E_{1}$ elliptic eigenmode at $k=3.5$, (b) $E_{2}$ elliptic eigenmode at $k=3.5$, (c) growth rate for $C=0.75$ and $\mathrm{Re}=400$ showing the existence of two distinct instability mode $E_{1}$ and $E_{2}$ with a crossover. The two modes that coexist for $k=3.5$ are plotted in (a) and (b) for the branch $E_{1}$ and $E_{2}$, respectively.

predicts that in the inviscid case, this configuration should be neutral $(\sigma=0)$.

\section{Core mode}

Since the symmetric and the anti-symmetric perturbations present similar evolution of the growth rate with $k$ and similar structures for the eigenmodes, here we will describe only the anti-symmetric case.

The eigenmodes associated with the band E1 are given in Fig. 9 for Reynolds number $\mathrm{Re}=5000$ and the three concentrations studied. We show only the perturbation relative to the base vortex of positive sign. The eigenmodes shown in Figs. 9(a) and 9(b) correspond to the wave numbers that result in the maximum growth rate $(k=4.7$ for $C=0.9$ and $k=2.9$ for $C=0.75$ ). For Fig. 9(c), since no maximum appears for the concentration $C=0.5$ at $\operatorname{Re}=5000$, we have chosen the wave number $k=1.6$ that gave the maximum growth rate at $\mathrm{Re}=400$. In each perturbation field presented in Fig. 9, the eigenmode is formed by two opposite sign 
spanwise vorticity perturbations nested inside the core of each vortex. This perturbation affects only the inner region of each vortex. It corresponds to a deformation of the inner core of each vortex and is not just a simple displacement of the vortex as a whole as was the case in the previous section.

The eigenmode corresponding to the band E2 is presented in Fig. 10(b) for the concentration $C=0.75$ and $\mathrm{Re}$ $=5000$. The transverse vorticity perturbations, more complex than in the band E1, are concentrated in two pairs of opposite sign perturbations, surrounded by two ring-like parts of opposite vorticity.

These perturbation structures obtained in the bands E1 and E2 are characteristic of the short wave bending instability of a flow with elliptic streamlines, whose radial eigenmodes become more complex as the wave number is increased. They have been described among others by Moore and Saffman, ${ }^{31}$ Tsai and Widnall, ${ }^{32}$ and Robinson and Saffman. ${ }^{33}$ The mechanism of this instability relies on a triadic interaction between the strain field and two Kelvin waves with azimuthal wave numbers $m=+1$ and $m=-1$ which resonate when they have the same frequency $\omega$. The most amplified instability modes are obtained for $\omega=0$. The eigenmodes obtained in band E1, Figs. 9 and 10(a), are similar to both the 3D mode measured by Leweke and Williamson $^{34}$ [Fig. 10(b)] in a vortex pair generated by a flap mechanism and the 3D mode found by Pierrehumbert (Fig. 2 of Ref. 11).

Observing the mode structure and the form of the growth rate curve as a function of $k$, one may wonder if the E1 and E2 modes are fundamentally distinct or if it is just one single mode which changes continuously with an increasingly complex core structure as $k$ increases. To answer this question, a Krylov method ${ }^{27,35}$ has been implemented in the present numerical code to estimate the two leading eigenvalues and eigenvectors. Equation (11) is integrated using the same numerical method (see Sec. III for details) until two distinct times $T$ and $T+\Delta T$, with $T$ and $\Delta T$ long periods of time. The vector $\mathbf{u}(T+\Delta T)$ is then orthogonalized to $\mathbf{u}(T)$ and both vectors are normalized resulting in vectors $\mathbf{v}_{1}$ and $\mathbf{v}_{2}$, respectively. The space spanned by $\left[\mathbf{v}_{1}, \mathbf{v}_{2}\right]$ is called the Krylov subspace. The linear operator $L$ constituted by the righthand-side terms of Eq. (11) are then estimated in the Krylov subspace to give $\left[\mathbf{v}_{1}^{\prime}, \mathbf{v}_{2}^{\prime}\right]$. The transfer matrix linking $\left[\mathbf{v}_{1}^{\prime}, \mathbf{v}_{2}^{\prime}\right]$ to $\left[\mathbf{v}_{1}, \mathbf{v}_{2}\right]$ is then diagonalized giving the two leading eigenvalues $\lambda_{i}$ and eigenvectors $\Phi_{i}$. The error made on the leading eigenvalues is evaluated by the ratio $\| L \Phi_{i}$ $-\lambda_{i} \Phi_{i}\|/\| \Phi_{i} \|$ and is less than $10^{-3}$ for both leading modes.

Figure 10(c) presents the growth rate of the first two elliptical modes E1 and E2 obtained for $C=0.75$ at $\mathrm{Re}$ $=5000$. Figures $10(\mathrm{a})$ and $10(\mathrm{~b})$ are the eigenfunctions corresponding to each instability branch at the same value of $k$, $k=3.5$ (points $e_{1}$ and $e_{2}$ ). It can be seen in Fig. 10(c) that curves corresponding to two elliptic modes E1 and E2 [Figs. 10(a) and 10(b)] cross. This indicates that E1 and E2 are independent instability modes. This same observation has also been reported by Klaassen and Peltier ${ }^{14}$ while studying the stability of a row of vortices of the same sign. However, it is possible that the numerical resolution accessible at that

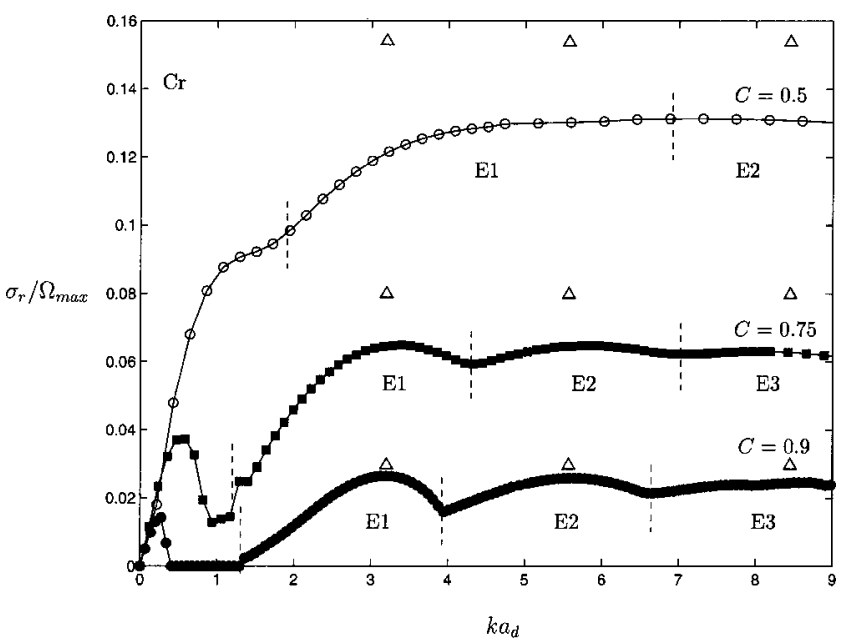

FIG. 11. Nondimensionalized growth rate curves of the anti-symmetric mode. $\Omega_{\max }$ is the maximum vorticity achieved at the vortex center and $a_{m}$ the vortex radius. Triangles correspond to Tsai and Widnall's and Robinson and Saffman's predictions.

time did not allow Pierrehumbert and Widnall ${ }^{10}$ to identify properly the different modes.

Tsai and Widnall, ${ }^{32}$ studying a single uniform vortex subjected to a weak strain field, have shown that the frequency scale of the elliptic instability is $\Omega_{\max }$ and the length scale is the core size. Robinson and Saffman ${ }^{33}$ have extended this study to large strain fields, confirming that the asymptotic predictions are applicable to finite strain. Since it is clear from the above arguments that this instability should be identified as a core instability, we have re-scaled the growth rate curves for the case of $\mathrm{Re}=5000$. As proposed by Tsai and Widnall, ${ }^{32}$ the wave number $k$ has been multiplied by the vortex radius $a$ and the growth rate $\sigma$ has been divided by $\Omega_{\max }$. Both quantities $a$ and $\Omega_{\max }$ were determined in Sec. II.

Figure 11 shows that the amplification maxima that we have calculated for $C=0.9$ and $C=0.75$ are achieved approximately for the same value $(k a)$. Moreover, this is true for the maxima of both bands E1 and E2. This means that the most amplified wave number in each band scales with the vortex core size. Furthermore, the maximum growth rate is smaller for $C=0.9$ than for $C=0.75$. All these features are consistent with Tsai and Widnall's predictions since resonance bands $E_{i}$ scale with $k a$ in their theory and the larger the elipticity parameter, the larger the growth rate. For the M\&M flow, the elipticity decreases when the concentration parameter $C$ increases (see Table I), and consequently so does the re-scaled growth rate (Fig. 11). All those trends are only qualitatively followed for the instability modes when $C=0.5$ since, as was already mentioned in the Sec. IV B 1 discussing the Crow instability, the vortices are not well separated for this low concentration parameter value and the analysis adapted for isolated vortices can only be expected to qualitatively predict the dynamics of this concentration.

Furthermore, Tsai's and Widnall's theory ${ }^{32}$ has been developed for a vortex with uniform vorticity while the vorticity in M\&M vortices is distributed, even when they are well separated. 
TABLE III. Table of the ellipticity $\varepsilon$ for the concentration parameters $C$ $=0.75$ and $C=0.9$.

\begin{tabular}{lll}
\hline \hline \multicolumn{1}{c}{$C$} & \multicolumn{1}{c}{$\theta$} & \multicolumn{1}{c}{$\varepsilon$} \\
\hline 0.9 & 1.11 & 0.052 \\
0.75 & 1.33 & 0.14 \\
0.5 & 2 & 0.26 \\
\hline \hline
\end{tabular}

We now compare quantitatively our results with Tsai and Widnall theory. Tsai and Widnall have shown that for the first two modes, bands $E_{1}$ and $E_{2}$, the most amplified nondimensional wave numbers are such that $k_{1} a=2.5$ and $k_{2} a$ $=4.35$. The corresponding nondimensional growth rates are $\sigma_{1} / \Omega_{0}=0.5708 \varepsilon, \sigma_{2} / \Omega_{0}=0.5695 \varepsilon$, with $\Omega_{0}$ the value of the uniform vorticity and $\varepsilon$ the ratio between the rate of strain and the vorticity. The parameter $\varepsilon$ may be computed following Robinson and Saffman ${ }^{21}$ using the aspect ratio $\theta$ of the ellipse defined in Sec. II:

$$
\varepsilon=2 \frac{\theta *(\theta-1)}{\left(\theta^{2}+1\right) *(\theta+1)} .
$$

The values of $\varepsilon$ are gathered in Table III.

Without any adjustment, estimating $\Omega_{0}$ by $\Omega_{\max }$, the prediction for the most amplified elliptic mode growth rate $\sigma_{\max }$ given by the asymptotic theory of Tsai and Widnall ${ }^{32}$ matches well with the numerically computed growth rate (see Fig. 11). The agreement is surprising since, the Tsai and Widnall model considers a single elliptic vortex with uniform vorticity whereas the vorticity is continuously distributed in Mallier and Maslowe model. If for $C=0.9$ it seems legitimate to consider that vortices are well separated the two vorticity distributions differ and only qualitative agreement would have been expected whereas quantitative agreement is obtained. For $C=0.5$ (and less obviously for $C=0.75$ ), the core size of the vortices is comparable to their spacing, therefore, coupling between vortices affects strongly the instability modes but still the Tsai and Widnall model predicts within a $20 \%$ accuracy the "growth rate."

While the vortex elipticity, defined uniquely from the Taylor expansion (6) of the vorticity in the center of the vortex, allows an accurate comparison of the growth rate with Tsai and Widnall's theory, ${ }^{32}$ it is not so for the vortex core size. Indeed, the value of the core size varies greatly according to the definition used to estimate it. There is no $a b$ initio theoretical reason to favor one definition over another when we want to compare the peak locations with the predictions of Tsai and Widnall ${ }^{32}$ since their basic state is a uniform vortex with a sharp boundary at $a=1$ whereas the present vortices possess a smooth vorticity field. This remark has already been made by Eloy and Le Dizès ${ }^{36}$ who have studied the elliptic instability of Gaussian vortices:

$$
\Omega(r)=\frac{\Gamma}{4 \pi \delta^{2}} e^{-r^{2} / 4 \delta^{2}},
$$

$\delta$ being a definition of the vortex core size. Eloy and Le Dizès $^{36}$ have shown that resonance occurs for $k_{1} \delta=1.13$ and $k_{2} \delta=1.97$, which differ from the Tsai and Widnall ${ }^{32}$ predictions.

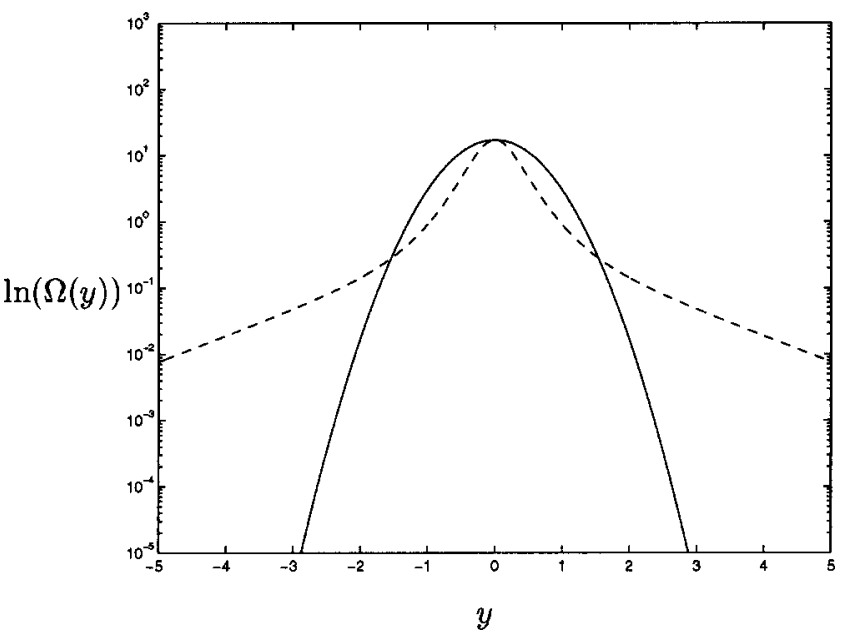

FIG. 12. Vorticity distribution at $x=0$ for a Gaussian vortex (full line) and the M\&M vortex for $C=0.9$ (dashed line).

In this study, we estimate the radius of the vortex by the first moment of the vorticity distribution (5) and if the vortex core size used in the Tsai and Widnall ${ }^{32}$ formula is empirically fitted as $a_{e}=0.8 a$ then, both for $C=0.75$ and $C=0.9$, the maxima of amplification for both E1 and E2 modes are well predicted (see Fig. 11). The fitting coefficient 0.8 expresses the adjustment from the core size estimated on the M\&M model to the core size estimated by a theory developed for a vortex with uniform vorticity. In the case of Gaussian vortices, the fitting coefficient that would allow comparison for the peak locations, using our definition of the core size (5) would be $a_{e}=1.2 a$. In the case of Gaussian vortices, $a$ underestimates the core size compared to Tsai and Widnall ${ }^{32}$ whereas in our model it overestimates it. This is not surprising since the M\&M solution relaxes very slowly to zero away from the core compared to Gaussian vortices (see Figs. 10 and 12).

Tsai and Widnall have predicted that the resonance band width of the amplified wave number varies proportionately to the ellipticity $\varepsilon$ and is equal to $(2.14 \varepsilon)$ for the first mode E1, and to $(3.5 \varepsilon)$ for the second one E2. Qualitatively, this trend is well recovered for the Mallier and Maslowe model since the curve for $C=0.9$, where the vortices are less elliptical, is sharper than for $C=0.75$. However, the "bells" have a larger and smoother top, even for $C=0.9$, whereas Tsai and Widnall predict a sharp peak. The smoothness of the vortex contour and the distribution of vorticity in the present study could explain the softness of the observed resonance.

\section{CONCLUSION}

We have investigated the 3D stability of an array of counter-rotating vortex pairs given by the Mallier and Maslowe model. The nature of the vorticity field of the base state depends on the value of the concentration parameter $C$. Three values have been investigated: $C=0.9$, approaching the limit of point vortices, $C=0.75$ characterizing vortices less concentrated but still separated, and $C=0.5$ representing large, smooth elliptical patches of vorticity. The perturbations considered are restricted to those with the streamwise 
periodicity of the base flow. The intrinsic symmetries of the base states with respect to the middle plane between two consecutive vortices allow us to distinguish two classes of perturbations, symmetric and anti-symmetric. For both classes, we have numerically determined the most unstable eigenmodes as a function of the spanwise wave number $k$ at Reynolds numbers $\operatorname{Re}=400$ and $\operatorname{Re}=5000$.

Depending on the symmetry and the wave number, we have identified three distinct instability mechanisms. At large wave numbers, the dominant mode corresponds to the elliptic instabilities of the vortex cores. In this case, symmetric and anti-symmetric modes were found to be equally amplified. Core instabilities induce a distortion of the inner part of the base vortices and the corresponding eigenmode becomes increasingly complex as the spanwise wave number is increased. Despite the periodicity and the vorticity distribution of the base state, a good quantitative agreement concerning the most amplified wave number and the corresponding growth rate has been found between the results obtained for concentration parameter $C=0.9$ and $C=0.75$ and the elliptic instability features of a uniform filament of vorticity predicted by Tsai and Widnall in the limit of infinitely small strain field. The most amplified wave number of these different core instabilities scales with the vortex core size and their maximum growth rate divided by the local maximum of vorticity scales with the ellipticity of the vortex.

At small wave numbers, $k<1$, the anti-symmetric mode exhibits an isolated peak corresponding to a Crow-type instability. The unstable wave number band and the corresponding maximum growth rate vary weakly with $C$. Comparison with the Crow instability is remarkably good for the three concentration parameters studied. The instability leads to an out-of-phase undulation of the base vortices in symmetric planes inclined at $45^{\circ}$ to the axis of the vortex.

For $2 \mathrm{D}$ perturbations, i.e., at $k=0$, the symmetric mode has been found to be unstable with a growth rate which depends on both the concentration parameter and the Reynolds number. This instability leads to the formation of two staggered rows of counter-rotating vortices, as previously identified by Dauxois et al. in their 2D linear stability study of the Mallier and Maslowe solution.

The present study should be applied with caution to the stability analysis of the wake of a bluff-body, since neither the thickness nor the hyperbolic regions of the vortex street are fully captured by the Mallier and Maslowe flow. Nevertheless, we can conclude that the Crow instability, scaling with the $2 \mathrm{D}$ wavelength $\lambda_{2 \mathrm{D}}$, should exist until the distance between the two rows equals $0.34 \lambda_{2 \mathrm{D}}$, a result predicted by Robinson and Saffman. Likewise, the elliptic modes should persist for staggered streets when the core of the vortex remains small comparing to $\lambda_{2 \mathrm{D}}$. However, a modification of the growth rate curves linked to a new selection is suspected to appear at large wave number $k$, due to the effect of hyperbolic regions of the type found in the Kelvin-Helmholtz street by Klaassen and Peltier.

\section{ACKNOWLEDGMENTS}

The authors would like to warmly thank Carlo Cossu, who contributed to work out the numerical Krylov method, and Laurette Tuckerman and Paul Billant for their thorough reading and their constructive observations.

${ }^{1}$ L. Rayleigh, Scientific Papers, Vol. 3 (Cambridge University Press, Cambridge, 1892).

${ }^{2}$ P. G. Drazin and W. H. Reid, Hydrodynamic Stability (Cambridge University Press, Cambridge, 1981).

${ }^{3}$ C. D. Winant and F. K. Browand, "Vortex pairing: the mechanism of turbulent mixing-layer growth at moderate Reynolds numbers," J. Fluid Mech. 63, 237 (1974).

${ }^{4}$ R. W. Miksad, "Experiments on the nonlinear stages of free shear layer transition,” J. Fluid Mech. 56, 695 (1972).

${ }^{5}$ J. H. Konrad, "An experimental investigation of mixing in twodimensional turbulent shear flows with applications to diffusion-limited chemical reaction," Internal Report No. CIT-8-PU, California Institute of Technology, Pasadena, 1976.

${ }^{6}$ F. R. Hama, "Three-dimensional vortex pattern behind a circular cylinder," J. Aeronaut. Sci. 24, 156 (1957).

${ }^{7}$ J. H. Gerrard, "The wakes of cylindrical bluff bodies at low Reynolds number," Philos. Trans. R. Soc. London, Ser. A 288, 351 (1978).

${ }^{8}$ A. J. Yule, "Large-scale structure in the mixing-layer of a round jet," J. Fluid Mech. 89, 413 (1978).

${ }^{9}$ P. A. Monkewitz, B. Lehmann, B. Barsikow, and D. W. Bechert, "The spreading of self-excited hot jets by side-jets," Phys. Fluids A 1, 446 (1989).

${ }^{10}$ R. T. Pierrehumbert and S. E. Widnall, "The two- and three-dimensional instabilities of a spatially periodic shear layer," J. Fluid Mech. 114, 59 (1982).

${ }^{11}$ R. T. Pierrehumbert, "Universal short-wave instability of two-dimensional eddies in an inviscid fluid," Phys. Rev. Lett. 57, 2157 (1986).

${ }^{12}$ B. J. Bayly, "Three-dimensional instability of elliptical flow," Phys. Rev. Lett. 57, 2160 (1986).

${ }^{13} \mathrm{~F}$. Waleffe, "On the three-dimensional instability of strained vortices," Phys. Fluids A 2, 76 (1990).

${ }^{14}$ G. P. Klaassen and W. R. Peltier, "The influence of stratification on secondary instability in free shear layers," J. Fluid Mech. 227, 71 (1991).

${ }^{15}$ S. J. Lin and G. M. Corcos, "The mixing layer: deterministic models of a turbulent flow. Part 3: The effect of plane strain on the dynamics of streamwise vortices," J. Fluid Mech. 141, 139 (1984).

${ }^{16}$ J. C. Lasheras and H. Choi, "Three-dimensional instability of a plane free shear layer. An experimental study of the formation and evolution of streamwise vortices," J. Fluid Mech. 189, 53 (1988).

${ }^{17}$ E. Meiburg and J. C. Lasheras, "Experimental and numerical investigation of the three-dimensional transition in plane wakes," J. Fluid Mech. 190, 1 (1987).

${ }^{18}$ J. C. Lasheras and E. Meiburg, "Three dimensional vorticity modes in the wake of a flat plate," Phys. Fluids A 2, 371 (1990).

${ }^{19}$ C. H. K. Williamson, "Three-dimensional wake transition," J. Fluid Mech. 328, 345 (1994).

${ }^{20}$ C. H. K. Williamson, "Vortex dynamics in the cylinder wake," Annu. Rev. Fluid Mech. 28, 477 (1996).

${ }^{21}$ A. C. Robinson and P. G. Saffman, "Three-dimensional stability of vortex arrays," J. Fluid Mech. 125, 411 (1982).

${ }^{22}$ S. C. Crow, "Stability theory for a pair of trailing vortices," AIAA J. 8, 2172 (1970).

${ }^{23}$ T. Dauxois, S. Fauve, and L. Tuckerman, "Stability of periodic arrays of vortices," Phys. Fluids 8, 487 (1996).

${ }^{24}$ R. Mallier and S. A. Maslowe, "A row of counter-rotating vortices," Phys. Fluids A 5, 1074 (1993).

${ }^{25}$ P. Tabeling, B. Perrin, and S. Fauve, "Instability of a linear array of forced vortices," Europhys. Lett. 3, 459 (1987).

${ }^{26}$ D. Fabre, C. Cossu, and L. Jacquin, "Spatio-temporal development of the long- and short-wave vortex pair instabilities," Phys. Fluids 12, 1247 (2000).

${ }^{27}$ L. S. Tuckerman, "Asymmetry and Hopf bifurcation in spherical Couette flow," Phys. Fluids 7, 80 (1995).

${ }^{28}$ P. Billant, P. Brancher, and J.-M. Chomaz, "Three-dimensional stability of a vortex pair,” Phys. Fluids 11, 2069 (1999). 
${ }^{29}$ M. J. Landman and P. G. Saffman, "The three-dimensional instability of strained vortices in a viscous fluid," Phys. Fluids 30, 2339 (1987).

${ }^{30}$ H. Lamb, Hydrodynamics (Cambridge University Press, Cambridge, 1932).

${ }^{31}$ D. W. Moore and P. G. Saffman, "The instability of a straight vortex filament in a strain field," Proc. R. Soc. London, Ser. A 346, 413 (1975).

${ }^{32} \mathrm{C}-\mathrm{Y}$. Tsai and S. E. Widnall, "The stability of short waves on a straight vortex filament in a weak externally imposed strain field," J. Fluid Mech. 73, 721 (1976)
${ }^{33}$ A. C. Robinson and P. G. Saffman, "Three-dimensional stability of an elliptical vortex in a straining field," J. Fluid Mech. 142, 451 (1984).

${ }^{34}$ T. Leweke and C. H. K. Williamson, "Three-dimensional instabilities in wake transition," Eur. J. Mech. B/Fluids 17, 571 (1998).

${ }^{35}$ W. S. Edwards, L. S. Tuckerman, R. A. Friesner, and D. C. Sorensen, "Krylov methods for the incompressible Navier-Stokes equations," J. Comput. Phys. 110, 82 (1994).

${ }^{36}$ C. Eloy and S. Le Dizès, "Three-dimensional instability of Burgers and Lamb-Oseen vortices in a strain field," J. Fluid Mech. 378, 145 (1999). 